\title{
Sitagliptin ameliorates ER stress in diabetic kidney disease through upregulation of SIRT1
}

Received 09 October 2020

Accepted 31 January 2021

Qunzi Zhang, Junjie Jia, Li He, Ying Fan*, Niansong Wang*

Department of Nephrology, Shanghai Jiao Tong University Affiliated Sixth People's Hospital, 600 Yishan Road, Shanghai, China

Abstract

Objectives: Endoplasmic reticulum (ER) stress plays a significant role in the progression of diabetic kidney disease (DKD), and dipeptidyl peptidase-4 (DPP4) inhibitors are widely used antihyperglycemic agents, exerting renal beneficial effects in DKD. Here, we investigated the role of DPP4 inhibitor Sitagliptin (Sita) in ER homeostasis in the kidneys of diabetic DBA2/J (D2) mice and in albumin-stimulated HK-2 cells. Methods and Results: ER stress was observed both in vivo and in vitro, as reflected by notably increased glucose-regulated protein of $78 \mathrm{kDa}$ (GRP78), CHOP, high phosphorylation of PERK ( $\mathrm{p}-\mathrm{PERK}$ ), and cleaved caspase3 (c-CASP3), whereas Sita effectively attenuated these disorders. Meanwhile, Sita increased the expression of SIRT1 both in vivo and in vitro. To further validate the potential effects of SIRT1 in regulating ER stress, we regulated SIRT1 by siRNA and overexpressed plasmids in albumin-overloaded HK-2 cells. Elevated SIRT1 alleviated albumin-induced ER stress, while decreased SIRT1 further aggravated ER stress in albumin-treated HK-2 cells. Conclusion: The results suggest that a novel mechanism links the DPP4 enzyme to ER stress during tubular injury in DKD and highlight that SIRT1 may be a potential target for managing DKD.

Keywords

diabetic kidney disease $\cdot$ ER stress $\bullet$ SIRT1 $・$ Sitagliptin $\bullet$ tubular injury

\section{Introduction}

Diabetic kidney disease (DKD) has become the leading cause of chronic kidney disease (CKD) [1]. Among the changes in the progression of DKD, renal tubular injury plays a critical role, which is more closely related to renal outcomes during end-stage renal disease (ESRD), presenting tubulointerstitial compartments with intrarenal vasculature thickening and hyalinization [2, 3]. Although the pathogenetic mechanism of diabetic tubular injury remains unclear, several studies have validated that cellular apoptosis largely contributes to its development $[4,5]$.

Accumulating evidence indicates that endoplasmic reticulum (ER) stress could be induced by several factors, such as hypoxia, hyperglycemia, reactive oxygen species (ROS), and proteinuria, which accelerate the progression of DKD. [6]. In DKD, a variety of stimulations break ER homeostasis, subsequently inducing unfolded protein response (UPR), and eventually activating the cell death pathway $[6,7]$.

Glucose-regulated protein of $78 \mathrm{kDa}$ (GRP78) serves as a central regulator of three main UPR sensors, namely, activating transcription factor (ATF6), inositol-requiring enzyme (IRE)-1 $\alpha$, and protein kinase RNA-like ER kinase (PERK), which initiate the UPR signaling pathway under ER stress [8]. Several past studies have shown that ER stress contributes to the development of CKD and DKD, while inhibition of the ER stress could restore the progression of various renal diseases $[9,10]$. In our past studies, we also found that inhibition of ER stress by tauroursodeoxycholic acid (TUDCA) significantly alleviated renal tubular injury and ER stressassociated cellular apoptosis in diabetic $\mathrm{db} / \mathrm{db}$ mice [7]. It is likely that elevated ER stress processes contribute to the renal functional deterioration and tubular injury that occur in the context of DKD, although the precise mechanism underlying the disruption of these processes remains unclear.

Dipeptidyl peptidase-4 (DPP4) inhibitors are widely used antihyperglycemic agents that prolong the effects of glucagon-like peptide-1 (GLP-1) and glucose-dependent insulinotropic polypeptide (GIP), which further stimulate the secretion of insulin [11-13]. Notably, DPP4 inhibitors exert positive renal effects in different renal diseases: Sitagliptin (Sita) alleviates kidney oxidative stress in STZ-induced diabetic rats, [14] and linagliptin shows obvious effects in preventing CKD progression in rats with 5/6 nephrectomy [15] and in GLP-1R knockout mice with CKD, [11] indicating that hypoglycemic effects may play a minor role underlying the beneficial renal effects of DPP4 inhibition. In our previous study, we found that Sita delays the progression of DKD in STZ-induced D2 mice, ameliorating mitochondrial abnormalities, preventing renal interstitial fibrosis, and attenuating tubular cell apoptosis [16]. However, whether DPP4 inhibitors regulated ER stress in diabetic STZ-induced mice remains unclear.

Sirtuin 1 (Sirt1), an $\mathrm{NAD}^{+}$-dependent protein deacetylase, plays multiple roles in cells involving hypoxic stress, DNA repair, cell senescence, inflammation, and mitochondrial regulation [17]; meanwhile, its degradation has essential 
effects on the development of DKD. In our past experiments, we found that SIRT1 was notably decreased in the kidneys of diabetic $\mathrm{db} / \mathrm{db}$ mice [18]. Recent studies, however, show that SIRT1 participates in the ER stress response related to hyperglycemia and hypoxia, [19-21] but whether SIRT1 is involved in the protection of DPP4 inhibitors is still unknown.

Therefore, our current study aimed to determine whether the DPP4 inhibitor Sita could ameliorate renal tubular injury in STZ-induced diabetic mice through inhibiting ER stress, and whether this protective effect is mediated by SIRT1. Our studies may provide additional mechanistic insights into the pathogenesis of DKD.

\section{Materials and Methods}

\subsection{Experimental animals and protocols}

Eight-week-old DBA2/J (D2) male mice were purchased from Beijing HFK Bioscience and housed under a constant 12-h light-dark cycle; they were free to eat and drink in the SPF room. D2 mice were randomly divided into three experimental groups $(n=8-12)$ : a control group, an STZ intraperitoneal injection group, and an STZ-treated group with Sita supplementation. Diabetic mice were induced by daily intraperitoneal injections of STZ $(50 \mathrm{mg} / \mathrm{kg}$ body wt.; SigmaAldrich) for 5 consecutive days. After 1 week of observation and recovery, the blood glucose levels were measured after $6 \mathrm{~h}$ of fasting. In this study, diabetic mice with fasting blood glucose exceeding $250 \mathrm{mg} / \mathrm{dl}$ for at least 2 consecutive days were considered to be successfully created.[5, 22] Diabetic mice were give Sita at $20 \mathrm{mg} / \mathrm{kg}$ (biovision chemical) daily intragastric administration, which was initiated 4 weeks after STZ stimulation and lasted 12 weeks. All animal procedures used in this study were approved by the Animal Care and Use Committee of Shanghai Jiao Tong University.

\subsection{Assessment of physiological features and renal func- tions}

The measurements of blood glucose and the collection of urine of these mice were carried out monthly. Blood glucose was measured by the Roche meter. Serum creatinine levels were measured through the Creatinine Assay Kit (BioAssay Systems, DICT-500) according to the manufacturer's instructions. Mice urine was collected over $24 \mathrm{~h}$ and urine creatinine and albumin levels were detected using ELISA kits (Abcam, ab204537 and ab108792). Urinary albumin/creatinine ratio (ACR) (in milligrams per gram) was calculated as follows: urine albumin (milligrams per decilitre)/urine creatinine (grams per decilitre).

\subsection{Hematoxylin-eosin (HE), PAS, and Masson staining}

For the morphometric studies, 3- $\mu m$-thick sections of paraffin-embedded tissues were stained with HE, PAS, and Masson solutions. To assess and calculate the renal histological damage, digital images of the glomeruli and tubules were obtained using light microscopy (original magnification 400x). A total of 20 images were randomly selected by two blinded researchers for quantitative and histochemical quantitation. The glomerular injuries were graded as follows: 0 represents no lesion; $1+$ represents sclerosis of $<25 \%$ of the glomerulus; and $2+, 3+$, and $4+$ represent sclerosis of $>25-50,>50-75$, and $>75 \%$ of the glomerulus [23]. Tubular injury was defined and calculated as follows: score 0: no tubular injury; score 1: $<10 \%$ of tubules injured; score $2: 10-25 \%$ of tubules injured; score 3: $25-50 \%$ of tubules injured; score $4: 50-74 \%$ of tubules injured; and score 5 : $>75 \%$ of tubules injured, as previously described $[24,25]$.

\subsection{Cell culture}

Conditionally immortalized human renal proximal tubular epithelial cell line (HK-2) was cultured in DMEM/F-12 containing $10 \%$ fetal bovine serum at $37^{\circ} \mathrm{C}$. We then treated $\mathrm{HK}-2$ cells with human serum albumin (HSA) at $20 \mathrm{mg} / \mathrm{ml}$ to mimic the injury caused by hyperglycemia or diabetic state.

To pharmacologically inhibit the DPP4 enzyme activity, cells were pretreated with Sita alone (5 $\mathrm{m}$, Biovision) for $4 \mathrm{~h}$, and then the cells were exposed to the culture medium with albumin and Sita for $72 \mathrm{~h}$. For gene disruption, Lipofectamine 3000 and P3000 reagents (Invitrogen, Grand Island, NY) were used to transfect cells according to the manufacturer's instructions at $60-70 \%$ confluent conditions. The cells were treated with SIRT1 siRNA (riboBio, Guangzhou, China), and the special sequences of Sirt1-siRNA were as follows: sense: 5'-CCAGUAGCACUAAUUCCAATT-3" and antisense: 5'-UUGGAAUUAGUGCCACUGGTT-3'. For overexpression of SIRT1, pCMV3.0 cDNA plasmids (Sino Biological, Beijing, China) were used according to the manufacturer's instructions.

\subsection{Western blot (WB) analysis}

Total protein was extracted from cells and renal tissues with radio immunoprecipitation assay (RIPA) lysis buffer (Beyotime Tech, Shanghai, China) with mixed inhibitors of. phosphatase and protease at $4^{\circ} \mathrm{C}$. The protein suspension was centrifuged at $14,000 \times \mathrm{g}$ and the medium containing total proteins was collected for further examination. For WB analysis, each sample containing $50 \mu \mathrm{g}$ protein, and the PVDF membrane was incubated overnight at $4^{\circ} \mathrm{C}$ with specific primary antibodies. The primary antibodies of GRP78, CHOP, p-PERK, PERK, c-CASP 3, and GAPDH were purchased from Cell Signaling Technology, and the primary antibodies of DPP4 and Sirt1 were purchased from ABcam. Protein expression was normalized to GAPDH expression.

\subsection{Statistical analysis}

The results were expressed as means \pm SEM. Comparison of the results between the two groups was evaluated by one-way 
analysis of variance (ANOVA). If any statistically significant difference was detected, post hoc comparisons were performed using the least significant difference test. Statistical analysis was performed with the SPSS (version 25.0) or GraphPad Prism (version 6.0) software, while $P<0.05$ was considered as statistically significant.

\section{Results}

\subsection{Sita-attenuated renal injury in STZ-injected DBA2/J mice}

Sita, the inhibitor of DPP4 enzyme, was gavage fed at $20 \mathrm{mg} / \mathrm{kg}$ daily to inhibit the activity of the DPP4 enzyme. Eight-week-old male D2 mice were randomly divided into three groups: control group, STZ-induced diabetes group, and Sitatreated diabetic group. Consistent with our previous studies, [16] compared with the control mice, the STZ-treated diabetic D2 mice had higher blood glucose levels, more obvious urinary ACRs, and more elevated serum creatinine levels, but reduced body weight (Figures 1A-D). However, the diabetic mice that received dietary Sita had failed to significantly reduce the blood glucose but had an improvement in body weight as well as notably lower urinary ACRs and serum creatinine levels compared to the STZ group (Figures 1A-D). Histologically, the STZ group mice had increased periodic acid-Schiff-positive tubular brush border staining, which is indicative of excretion of proteins, notable renal tubular dilatation, hypertrophy of glomeruli, mesangial matrix deposition, and tubulointerstitial fibrosis as determined by $\mathrm{HE}, \mathrm{PAS}$, and Masson staining when compared with the control mice (Figures 1E-G). However, the STZ-treated mice with drug treatment showed notable improvement, largely in the tubular compartment with less cellular disruption; Sita administration also significantly ameliorated these morphologic injuries and tubular changes in STZ-induced diabetic mice (Figures 1E-G). Quantitative analyses with damage scores ranging from 0 to 3 indicated that STZ mice with Sita supplementation had slightly reduced glomerular damage but a notable decrease in tubular damage compared with the STZ group (Figures $1 \mathrm{H}, \mathrm{I}$ )

\subsection{Sita inhibited ER stress in the kidneys of STZ-in- duced diabetic mice}

To further determine whether the renal beneficial effects of Sita in STZ-induced diabetic mice were through ER stress, we examined the expression of ER stress-associated markers in the kidneys by WB analysis. Compared with the kidneys from control mice, the kidneys of STZ mice presented more increased phosphorylation of PERK at the Thr980 site (p-PERK), and higher expressions of GRP78 and CHOP (Figures 2A,B). However, all these elevations of ER stress-associated markers in diabetic mice were significantly alleviated by Sita supplementation (Figures 2A,B). These results reveal that Sita effectively alleviated the obvious ER stress in the kidneys of STZ-induced diabetic mice.

\subsection{Sita alleviated ER stress-associated apoptosis and upregulating SIRT1}

It has been reported that excessive elevation of the DPP4 enzyme may lead to the progression of DKD, in which SIRT1 may be involved [26-28]. Thus, we further determined whether the protective roles of Sita in the kidneys of diabetic mice were likely related to the SIRT1. First, we found that the protein expression of DPP4 in the kidneys was obviously increased in the STZ-induced diabetic mice compared with the control mice, and this increase was notably suppressed after Sita treatment (Figures 3A,B). In contrast, SIRT1 was significantly decreased in the kidneys of diabetic mice compared with those of control mice, as measured by WB, while these reductions were attenuated by Sita treatment (Figures 3A,B). However, further in vitro experiments are needed to clarify the relationship between ER stress and SIRT1 in the progression of DKD.

Given that Sita exerts nephroprotective effects on diabetic STZ mice, the apoptosis-related protein was also determined. Compared with the control mice, diabetic mice presented more elevated c-CASP3 in the renal lysates, while this disorder was effectively attenuated by Sita (Figures $3 \mathbf{A}, \mathbf{B}$ ). Collectively, these in vivo data suggest that Sita gives effective protection in the kidneys of STZ-induced diabetic mice through maintaining the ER stress, while SIRT1 may also be involved in these effects.

\subsection{Sita-alleviated ER stress in albumin-overloaded HK-2 cells}

Since our animal experiments suggest that Sita had a significant protective effect on the kidneys of diabetic mice, we identified the potential mechanism of this effect in vitro via HK-2 cells. In our previous study, it was confirmed that exposure of HK-2 cells to high protein load in the glomerular filtrate induces ER stress and cell apoptosis; [5, 16] therefore, in vitro, we used a high protein overload mode by exposing HK2 cells with endotoxin-free HSA $(20 \mathrm{mg} / \mathrm{ml})$. We determined whether Sita inhibits ER stress and delays cell apoptosis in HSA-induced HK2 cells. Compared with the control cells, HSA-treated HK-2 cells had notable improvement in the ER stress-associated markers and apoptotic proteins, showing more increased p-PERK, and highly elevated expressions of GRP78, CHOP, and c-CASP3, while these changes were attenuated after Sita treatment $(5 \mu \mathrm{m})$ (Figures $4 \mathrm{~A}-\mathrm{C})$. These results indicate that Sita had a positive effect on albumin-treated HK-2 cells through inhibiting the ER stress.

\subsection{Sita increased SIRT1 in albumin-overloaded HK-2 cells}

Then, we further examine the potential reason underlying its protective effects. Consistent with our in vivo data, Sita remarkably attenuated the elevated DPP4 expression in HK-2 cells stimulated by albumin (Figures 4D,E). Meanwhile, HSA notably decreased the expression of SIRT1, which were effectively restored by Sita (Figures 4D,E). Hence, these results indicate that Sita blocked HSA-activated ER stress 

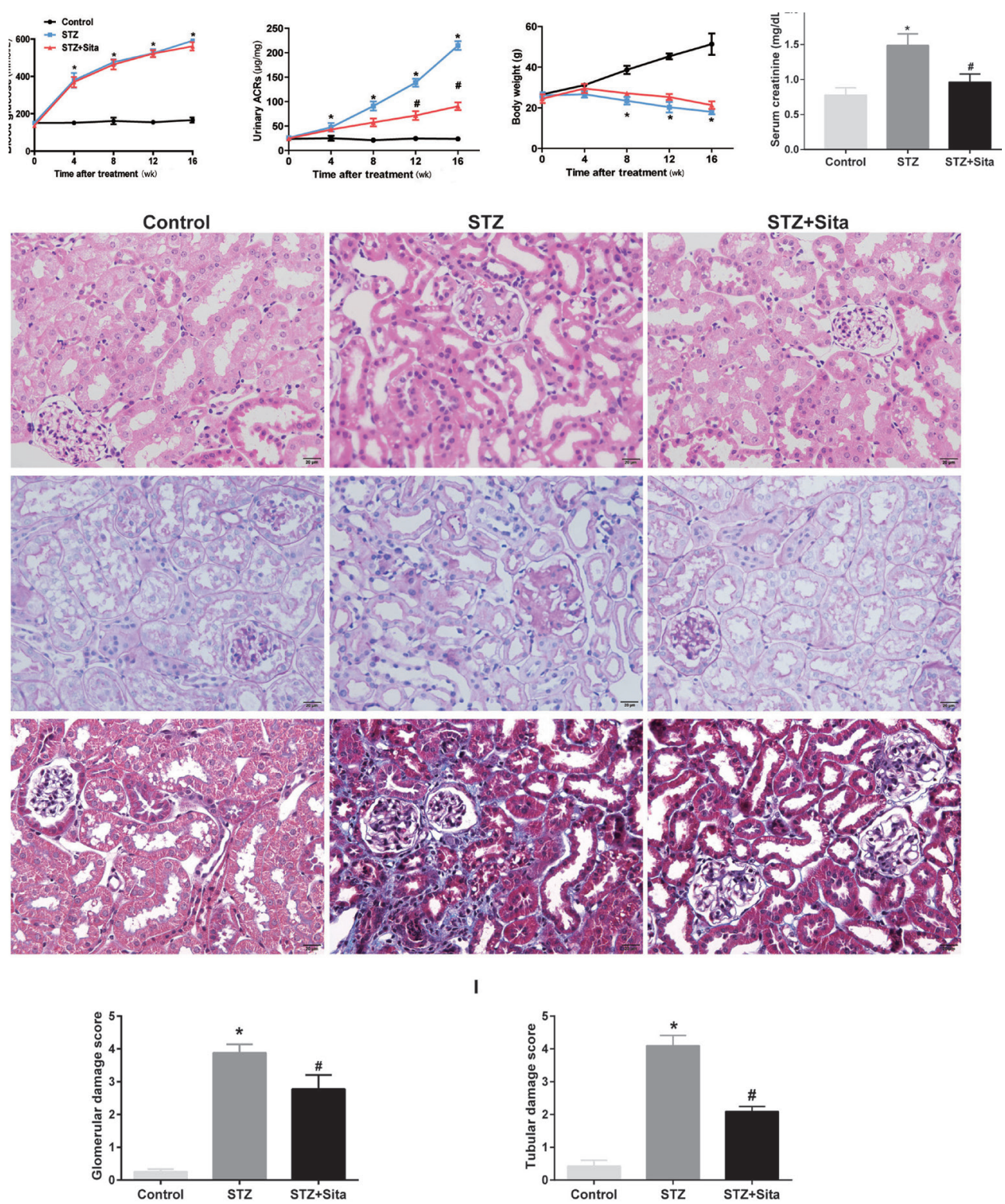

Figure 1. Renal tubular injury in diabetic mice was attenuated by Sita treatment. (A) Blood glucose levels, (B) urinary ACRs, (C) body weight, and (D) serum creatinine levels were examined in different groups. (E) Renal histology evaluations of different groups were performed with HE staining, (F) PAS staining, and (G) extracellular matrix accumulation and collagen fiber deposition were determined with Masson staining in different groups (original magnification 400x, bars $=20 \mu \mathrm{m}$ ). (H,I) Quantitative analyses with damage scores ranging from zero 0 to five 5 indicated that Sita treatment ameliorated the kidney changes after STZ induction of diabetes. Results are presented as means \pm SEM. $n=10$ for each group. $P$ as determined by ANOVA: ${ }^{*} P<0.05$ compared with the control group and \#P< 0.05 compared with the STZ group. Control, nondiabetic mice with saline gavage; STZ, STZ-treated diabetic mice with saline gavage; STZ+Sita, STZ-induced diabetic mice with Sita administration.

and upregulated SIRT1 in tubular cells through inhibiting the DPP4 enzyme.

\subsection{Downregulation of SIRT1-aggravated ER stress, whereas upregulation of SIRT1-attenuated ER stress markers in HSA-treated HK-2 cells}

To validate the role of SIRT1 in albumin-overloaded ER stress, we further confirmed whether regulation of SIRT1 influences imbalanced ER stress in HSA-treated HK-2 cells through using SIRT1 cDNA plasmids (oxp-SIRT1) and
SIRT1 siRNA. Compared with the control cells, si-SIRT1 in HK-2 cells yielded significantly decreased SIRT1 expression, higher p-PERK, and elevated expressions of GRP78 and $\mathrm{CHOP}$ (Figures $5 \mathrm{~A}, \mathbf{B}$ ), demonstrating the further activated ER stress. In contrast, SIRT1 overexpression notably attenuated HSA-stimulated abnormalities in the ER stress of HK-2 cells, as reflected by the decreased ER stress-associated markers ( $p$-PERK, GRP78, and CHOP) (Figures 5A,B). Thus, these results suggest that SIRT1 may be directly involved in the ER stress induced by albumin overload in the HK-2 cells. 


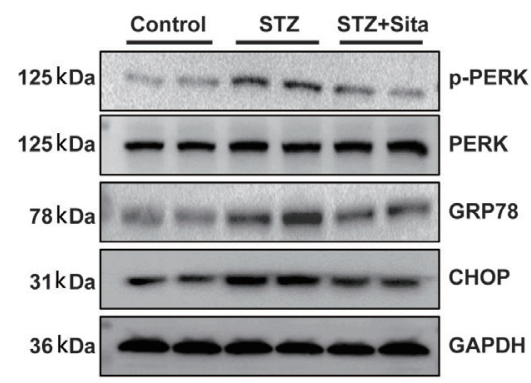

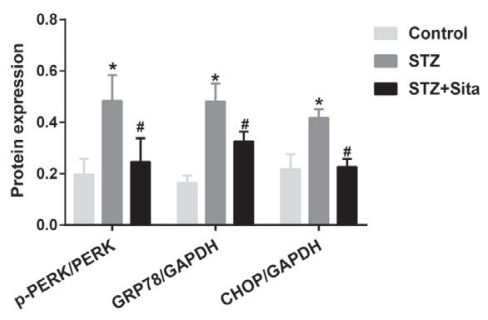

Figure 2. Sita restored the elevated ER stress in the kidneys of diabetic mice. $(A, B)$ Quantitative analysis and representative photographs of p-PERK (Thr980), PERK, CHOP, and GRP78 in different groups. Results are presented means \pm SEM. $n=10$ for each group. $P$ as determined by ANOVA: ${ }^{*} P<0.05$ compared with the control group and ${ }^{\#} P<0.05$ compared with the STZ group.

A

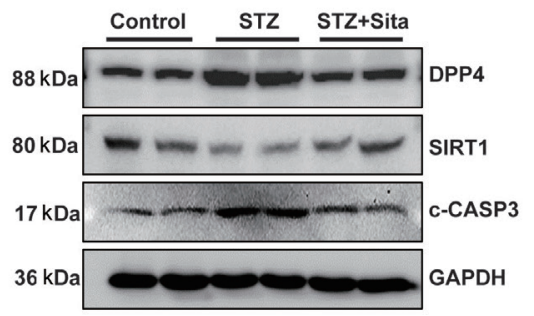

B

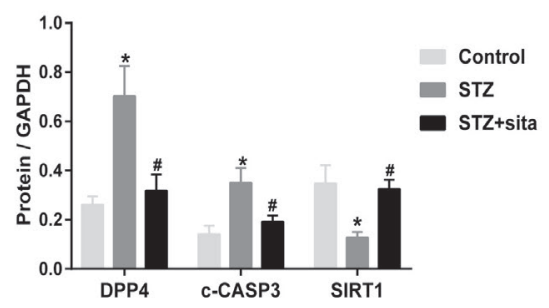

Figure 3. Sita reversed ER stress-related apoptosis and elevated SIRT1 in the kidneys of diabetic mice. (A,B) Quantitative analysis and representative photographs show the protein expression of DPP4, SIRT1a and C-CASP3 in different groups. Results are presented means \pm SEM. $n=10$ for each group. $P$ as determined by ANOVA: ${ }^{*} P<0.05$ compared with the control group and ${ }^{\#} P<0.05$ compared with the STZ group.

\section{Discussion}

\subsection{ER stress in the progression of DKD}

Renal tubulointerstitial fibrosis is the most common lesion of tubular injuries, some studies have reported that tubulointerstitial fibrosis appears earlier than glomerular fibrosis and is more closely related to renal outcomes during DKD progression [29]. Thus, it is important to understand the exact mechanism of tubular injury to develop specific therapies for diabetic tubular changes. However, in animal studies, it is difficult to successfully create significant tubular damages in diabetic models. Recent studies show that D2 mice may have a diabetes-susceptible background [23], which was confirmed in our past studies. In our previous work, tubular injuries and tubulointerstitial fibrosis were observed in STZinduced D2 mice with unilateral nephrectomy [16], which was significantly reversed by Sita administration. Thus, in our present study, we used STZ-injected D2 mice to study the potential mechanism of the DPP4 inhibitor Sita on tubular cell injury and abnormal ER stress of DKD.

ER stress exerts significant effects in the progression of DKD, while inhibition of ER stress through TUDCA presented obvious renoprotection in diabetic $\mathrm{db} / \mathrm{db}$ mice $[5,7]$. Proteinuria is a critical player in DKD and is positively correlated with the progression to ESRD [30, 31]. It has been reported that the exposure of proximal tubular epithelial cells to excess albumin causes renal tubular fibrosis in the progression of DKD [31], thus we use HSA-treated HK-2 cells to mimic the diabetic renal states. In our current work, we observed highly pronounced ER stress and its associated cellular apoptosis in the kidneys of STZ-diabetic D2 mice and HSA-treated HK-2 cells, showing the elevated expressions of GRP78, CHOP, p-PERK, and c-caspase3, which were consistent with our past studies in $\mathrm{db} / \mathrm{db}$ mice [7].

\subsection{Inhibition of the DPP4 inhibitor Sita on ER stress}

Recently, several new drugs have been approved for the treatment of T2DM, including DPP4 inhibitors. Recent studies also found that DPP4 inhibitors play beneficial effects on nondiabetic renal diseases, [32, 33] but the protective mechanism is still not well defined so far. In our study, we confirmed that Sita, a DPP4 inhibitor, improved renal functions and alleviated renal histological changes in the kidneys of STZ-treated diabetic mice without exerting hypoglycemic effects. The finding that Sita failed to exert 
A

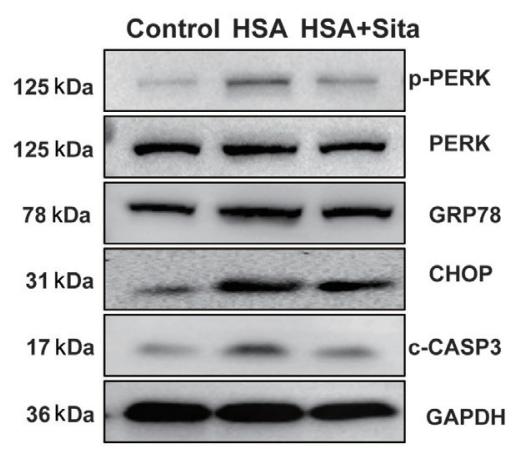

D

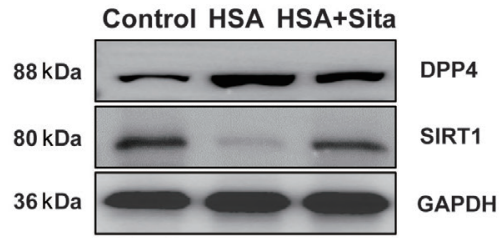

B

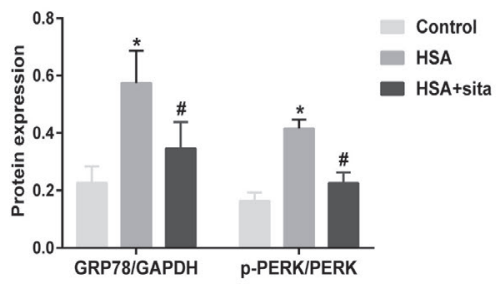

C

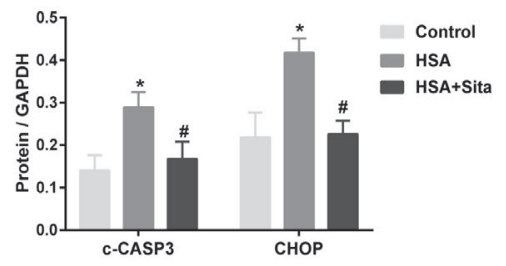

E

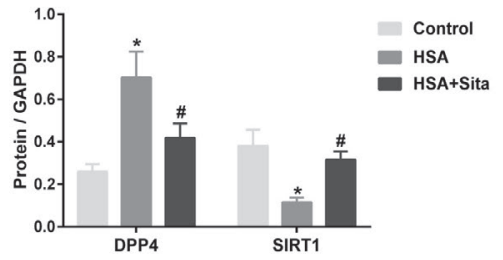

Figure 4. Sita restored the elevated ER stress-associated markers and apoptotic proteins, and improved SIRT1 in HSA-treated HK-2 cells. (AC) WB analysis and quantification analysis show the expression of ER stress-associated markers (p-PERK, GRP78) and apoptotic proteins (c-CASP3 and CHOP) in different groups. $(D, E)$ WB analysis and quantification analysis of the expressions of DPP4 and SIRT1 from different groups. HSA+Sita, Sita $(5 \mu \mathrm{m})$ was used to pretreat cells for $4 \mathrm{~h}$, and then HSA (20 mg/mL) was added and incubated for $72 \mathrm{~h}$. Results are presented means \pm SEM. $n=5$ for each group. $P$ as determined by ANOVA: * $P<0.05$ compared with the control group and ${ }^{\#}<0.05$ compared with the HSA group.

A

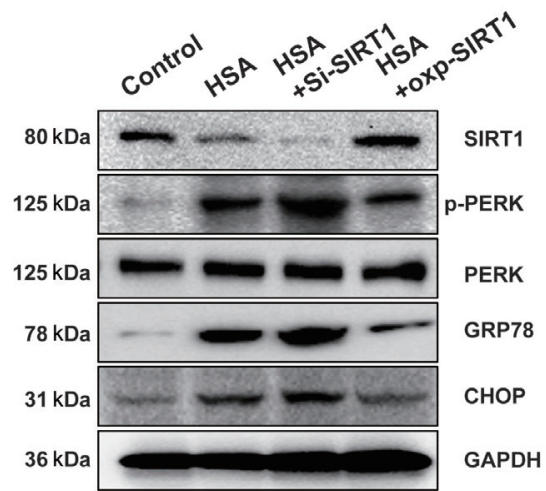

B

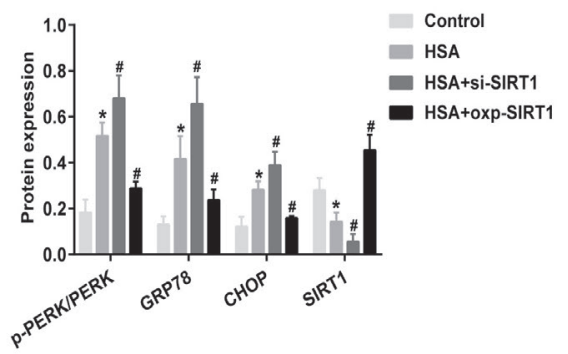

Figure 5. SIRT1 overexpression attenuated ER stress, while downregulated SIRT1 further accentuated ER stress in HSA-treated HK-2 cells. $(A, B) W B$ analysis and quantification analysis show the expression of SIRT1, p-PERK, GRP78, and CHOP in different groups. In the HSA+oxp-SIRT1 groups, SIRT1 was overexpressed by pcDNA3.0 (oxp-SIRT1), and the cells were then exposed to the albumin state $(20 \mathrm{mg} / \mathrm{mL})$ for $72 \mathrm{~h}$. In the HSA+si-SIRT1 groups, SIRT1 was decreased by siRNA sequence (si-SIRT1) via lipo3000, and then the cells were exposed to HSA for $72 \mathrm{~h}$. Results are presented means \pm SEM. $n=5$ for each group. $P$ was determined by ANOVA: ${ }^{*} P<0.05$ compared with the control group and ${ }^{\#} P<0.05$ compared with the HSA group.

a hypoglycemic role in STZ-diabetic mice is not surprising, as its effects are largely induced by activating GLP-1R, GIP, or other possible pathways that promote insulin secretion [27, 34]. However, the STZ-injected diabetic mice that we used are insulin-deficient mouse models [23], as STZ destroys the function of islets.
In this study, we found that ER stress-associated markers were ameliorated after Sita treatment in both diabetic mouse kidneys and HSA-treated HK-2 cells, as reflected by the decreased CHOP, GRP78, and p-PERK. Besides, ER stress-related apoptosis was also ameliorated by Sita, showing attenuated levels of cleaved-caspase 3 .The results provide evidence that 
the DPP4-mediated signaling pathway may partly be associated with ER stress-induced injury in diabetic tubules, but the potential mechanism underlying it is still unclear.

\subsection{Potential mechanism of renal beneficial effects of Sita in DKD progression}

Recent studies have focused on SIRT1, a deacetylase that involves various signaling pathways, including cell repair and mitochondrial function [35-37]. Recent studies also confirm its beneficial effects in managing DKD; [38] for example, upregulation of SIRT1 protects cells from apoptosis by inhibiting ER stress in hepatocytes and pancreatic $\beta$ cells; [39] SIRT1 also improved palmitate-induced insulin resistance via regulation of ER stress in HepG2 cells [40]. In our work, we demonstrated that SIRT1 expression was significantly decreased, whereas Sita treatment can effectively restore its expression. We further investigated the possibility that SIRT1 regulates ER stress in HSA-treated HK-2 cells via using the overexpressed SIRT1 plasmids and SIRT1 siRNA. The increased SIRT1 alleviated ER stress-associated markers and cellular apoptosis induced by albumin overloading in HK-2 cells, indicating that SIRT1 inhibited ER stress in the HSA state. Furthermore, si-SIRT1 confirmed the link between SIRT1, increased expression of c-CASP3, and activation of ER stress. These results are in agreement with previous studies, [41-44] which described the renal beneficial effects and anti-ER stress of SIRT1.

However, how DPP4-mediated the changes in SIRT1 and regulated its downstream ER stress and its exact role in the tubular cell injury has not been fully studied. In our study, we confirmed the destruction of ER homeostasis of HK-2 cells induced by overloaded albumin, and these effects were significantly attenuated by Sita treatment. Sita preserved the expression of SIRT1, and the downregulation of SIRT1 in HK-2 cells enhanced the HSA-induced ER stress in HK-2 cells, suggesting that SIRT1 may be regulated by DPP4.

There are several limitations in our work. This current work studied the beneficial effects induced by Sita via inhibiting ER stress, but we cannot exclude the possibility that these effects may also be attributed to other substrates of the DPP4 enzyme, given that the DPP4 enzyme cleaves a large number of chemokines, neuropeptides, and peptide hormones, like SDF-1a, substance P, peptide YY, and neuropeptide $Y$ [45]. We have studied the role of SDF-1 $\alpha$, a substrate of the DPP4 enzyme, in maintaining mitochondrial dynamic homeostasis in DKD [16]. The effects of these substrates on DKD progression are still unclear, and further investigations are needed to address these pathways individually and in detail. Another limitation of our study is that the study design is not usually comparable to the clinical conditions.

Collectively, in our present study, we found that the DPP4 inhibitor Sita prevented ER stress in STZ-induced diabetic D2 mice and HSA-treated HK-2 cells, whose protective effects may be through upregulating SIRT1. Thus, our results demonstrate a novel mechanism linking the DPP4 enzyme to ER stress during tubular injury in DKD.

\section{Acknowledgments}

This study was supported by the National Nature Science Foundation of China (81670657,81870504,81870468,81400735), National "Twelfth Five-Year" Plan for Science \& Technology Support (2013BAI02B01-01), Medical and Engineering Cross funding of Shanghai Jiao Tong University (YG2017MS10), and Open Project of Shanghai Key Laboratory of Sleep Disordered Breathing (SHKSDB-KF-19-04).

\section{Author Contributions}

Y.F., N.W., and Q.Z. designed the research project; Q.Z. and J.J. performed the experiments; Q.Z. and L.H. analyzed the renal histopathology; Q.Z., L.H., J.J., Y.W., and Y.F. analyzed the data; Q.Z. drafted the manuscript; and Y.F. and N.W. edited and revised the manuscript and approved the final version of the manuscript.

Conflicts of Interest

Niansong Wang is an Editorial Board Member of the journal. No other potential conflicts of interest relevant to this article are reported.

\section{REFERENCES}

[1] Kanwar YS, Sun L, Xie P, Liu FY, Chen S. A glimpse of various pathogenetic mechanisms of diabetic nephropathy. Annu Rev Pathol $2011 ; 6: 395-23$

[2] Bonventre JV. Can we target tubular damage to prevent renal function decline in diabetes? Semin Nephrol 2012; 32:452-62.
[3] Xiao L, Xu X, Xu Y, Zhang F, Wang M, Wang J, et al. The mitochondria-targeted antioxidant MitoQ ameliorated tubular injury mediated by mitophagy in diabetic kidney disease via Nrf2/PINK1. Redox Biol 2017; 11:297-11.

[4] Coughlan MT, Higgins GC, Nguyen TV, Penfold SA, Thallas-Bonke 
V, Tan SM, et al. Deficiency in apoptosis-inducing factor recapitulates chronic kidney disease via aberrant mitochondrial homeostasis Diabetes 2016; 65:1085-98.

[5] Fan Y, Xiao W, Li Z, Li X, Chuang PY, Jim B, et al. RTN1 mediates progression of kidney disease by inducing ER stress. Nat Commun 2015; 6:7841.

[6] Jia Y, Zheng Z, Yang Y, Zou M, Li J, Wang L, et al. MiR-4756 promotes albumin-induced renal tubular epithelial cell epithelial-to-mesenchymal transition and endoplasmic reticulum stress via targeting Sestrin2. J Cell Physiol 2019; 234:2905-15

[7] Ye Z, Zhang Q, Li Y, Wang C, Zhang J, Ma X, et al. High prevalence of hyperhomocysteinemia and its association with target organ damage in Chinese patients with chronic kidney disease. Nutrients 2016; 8:645.

[8] Liu X, Zhang R, Huang L, Zheng Z, Vlassara H, Striker G, et al. Excessive oxidative stress contributes to increased acute ER stress kidney injury in aged mice. Oxid Med Cell Longev 2019; 2019:2746521.

[9] Maekawa H, Inagi R. Stress signal network between hypoxia and ER stress in chronic kidney disease. Front Physiol 2017; 8:74.

[10] Allison SJ. Genetic kidney disease: Uromodulin in ER stress and apoptosis. Nat Rev Nephrol 2017; 13:722.

[11] Hasan AA, von Websky K, Reichetzeder C, Tsuprykov O, Gaballa MMS, Guo J, et al. Mechanisms of GLP-1 receptor-independent renoprotective effects of the dipeptidyl peptidase type 4 inhibitor linagliptin in GLP-1 receptor knockout mice with 5/6 nephrectomy. Kidney Int 2019; 95:1373-88.

[12] Gupta S, Sen U. More than just an enzyme: Dipeptidyl peptidase-4 (DPP-4) and its association with diabetic kidney remodelling. Pharmacol Res 2019; 147:104391.

[13] Takada S, Masaki Y, Kinugawa S, Matsumoto J, Furihata T, Mizushima W, et al. Dipeptidyl peptidase-4 inhibitor improved exercise capacity and mitochondrial biogenesis in mice with heart failure via activation of glucagon-like peptide-1 receptor signalling. Cardiovasc Res 2016; 111:338-47.

[14] Marques C, Goncalves A, Pereira PMR, Almeida D, Martins B, Fontes-Ribeiro C, et al. The dipeptidyl peptidase 4 inhibitor Sitagliptin improves oxidative stress and ameliorates glomerular lesions in a rat model of type 1 diabetes. Life Sci 2019; 234:116738.

[15] Tsuprykov O, Ando R, Reichetzeder C, von Websky K, Antonenko $\mathrm{V}$, Sharkovska $Y$, et al. The dipeptidyl peptidase inhibitor linagliptin and the angiotensin II receptor blocker telmisartan show renal benefit by different pathways in rats with 5/6 nephrectomy. Kidney Int 2016; 89:1049-61.

[16] Zhang Q, He L, Dong Y, Fei Y, Wen J, Li X, et al. Sitagliptin ameliorates renal tubular injury in diabetic kidney disease via STAT3dependent mitochondrial homeostasis through SDF-1alpha/CXCR4 pathway. FASEB J 2020; 34:7500-19.

[17] Ryu DR, Yu MR, Kong KH, Kim H, Kwon SH, Jeon JS, et al. Sirt1-hypoxia-inducible factor-1alpha interaction is a key mediator of tubulointerstitial damage in the aged kidney. Aging Cell 2019; 18:e12904.

[18] Zhang Q, Deng Q, Zhang J, Ke J, Zhu Y, Wen RW, et al. Activation of the Nrf2-ARE pathway ameliorates hyperglycemia-mediated mitochondrial dysfunction in podocytes partly through Sirt1. Cell Physiol Biochem 2018; 48:1-15.

[19] Kim JY, Jo J, Kim K, An HJ, Gwon MG, Gu H, et al.
Pharmacological activation of Sirt1 ameliorates cisplatin-induced acute kidney injury by suppressing apoptosis, oxidative stress, and inflammation in mice. Antioxidants (Basel) 2019; 8:322.

[20]. Chuang PY, Cai W, Li X, Fang L, Xu J, Yacoub R, et al. Reduction in podocyte SIRT1 accelerates kidney injury in aging mice. Am J Physiol Renal Physiol 2017; 313:F621-28.

[21] Papadimitriou A, Silva KC, Peixoto EBMI, Borges CM, de Faria $\mathrm{JML}$, de Faria JBL. Theobromine increases $\mathrm{NAD}(+) /$ Sirt- 1 activity and protects the kidney under diabetic conditions. Am J Physiol Renal Physiol 2015; 308:F209-25.

[22] Long M, Cai L, Li W, Zhang L, Guo S, Zhang R, et al. DPP-4 inhibitors improve diabetic wound healing via direct and indirect promotion of epithelial-mesenchymal transition and reduction of scarring. Diabetes 2018; 67:518-31.

[23] Qi H, Casalena G, Shi S, Yu L, Ebefors K, Sun Y, et al. Glomerular endothelial mitochondrial dysfunction is essential and characteristic of diabetic kidney disease susceptibility. Diabetes 2017; 66:763-78. [24] Chen J, Chen JK, Conway EM, Harris RC. Survivin mediates renal proximal tubule recovery from AKI. J Am Soc Nephrol 2013; 24:2023-33.

[25] Dong Y, Zhang Q, Wen J, Chen T, He L, Wang Y, et al. Ischemic duration and frequency determines AKI-to-CKD progression monitored by dynamic changes of tubular biomarkers in IRI mice. Front Physiol 2019; 10:153.

[26] Mi DH, Fang HJ, Zheng GH, Liang XH, Ding YR, Liu X, et al. DPP-4 inhibitors promote proliferation and migration of rat brain microvascular endothelial cells under hypoxic/high-glucose conditions, potentially through the SIRT1/HIF-1/VEGF pathway. CNS Neurosci Ther 2019; 25:323-32.

[27] Nistala R, Savin V. Diabetes, hypertension, and chronic kidney disease progression: Role of DPP4. Am J Physiol Renal Physiol 2017; 312:F661-70.

[28] Long Z, Cao M, Su S, Wu G, Meng F, Wu H, et al. Inhibition of hepatocyte nuclear factor $1 \mathrm{~b}$ induces hepatic steatosis through DPP4/NOX1-mediated regulation of superoxide. Free Radical Bio Med 2017; 113:71-83.

[29] Bonventre JV. Can we target tubular damage to prevent renal function decline in diabetes? Semin Nephrol 2012; 32:452-62.

[30] Lindenmeyer MT, Rastaldi MP, Ikehata M, Neusser MA, Kretzler $\mathrm{M}$, Cohen $\mathrm{CD}$, et al. Proteinuria and hyperglycemia induce endoplasmic reticulum stress. J Am Soc Nephrol 2008; 19:2225-36.

[31] Nordquist L, Friederich-Persson M, Fasching A, Liss P, Shoji K, Nangaku $\mathrm{M}$, et al. Activation of hypoxia-inducible factors prevents diabetic nephropathy. J Am Soc Nephrol 2015; 26:328-38.

[32] Chang YP, Sun B, Han Z, Han F, Hu SL, Li XY, et al. Saxagliptin attenuates albuminuria by inhibiting podocyte epithelial- to-mesenchymal transition via SDF-1a in diabetic nephropathy. Front Pharmacol 2017; 8:780.

[33] Hasan AA, Hocher B. Role of soluble and membrane-bound dipeptidyl peptidase-4 in diabetic nephropathy. J Mol Endocrinol 2017; 59:R1-10.

[34] Groop PH, Cooper ME, Perkovic V, Emser A, Woerle HJ, von Eynatten M. Linagliptin lowers albuminuria on top of recommended standard treatment in patients with type 2 diabetes and renal dysfunction. Diabetes Care 2013; 36:3460-68.

[35] Guan Y, Hao CM. SIRT1 and kidney function. Kidney Dis (Basel) 2016; 1:258-65. 
[36] Nguyen LT, Mak CH, Chen H, Zaky AA, Wong MG, Pollock CA, et al. SIRT1 attenuates kidney disorders in male offspring due to maternal high-fat diet. Nutrients 2019; 11:146.

[37] Ryu DR, Yu MR, Kong KH, Kim H, Kwon SH, Jeon JS, et al. Sirt1hypoxia-inducible factor-1 $\alpha$ interaction is a key mediator of tubulointerstitial damage in the aged kidney. Aging Cell 2019; 18:e12904.

[38] Zhong Y, Lee K, He JC. SIRT1 is a potential drug target for treatment of diabetic kidney disease. Front Endocrinol (Lausanne) 2018; 9:624.

[39] Wang FM, Chen YJ, Ouyang HJ. Regulation of unfolded protein response modulator $\mathrm{XBP} 1 \mathrm{~s}$ by acetylation and deacetylation. Biochem J 2011; 433:245-52.

[40] Jung TW, Lee KT, Lee MW, Ka KH. SIRT1 attenuates palmitate-induced endoplasmic reticulum stress and insulin resistance in HepG2 cells via induction of oxygen-regulated protein 150. Biochem Biophys Res Commun 2012; 422:229-32.

[41] Chuang PY, Cai W, Li X, Fang L, Xu J, Yacoub R, et al. Reduction in podocyte SIRT1 accelerates kidney injury in aging mice. Am J
Physiol Renal Physiol 2017; 313:F621-28.

[42] Kim JY, Jo J, Kim K, An HJ, Gwon MG, Gu H, et al. Pharmacological activation of Sirt1 ameliorates cisplatin-induced acute kidney injury by suppressing apoptosis, oxidative stress, and inflammation in mice. Antioxidants (Basel) 2019; 8:322.

[43] Wang X, Liu R, Zhang W, Hyink DP, Das GC, Das B, et al. Role of SIRT1 in HIV-associated kidney disease. Am J Physiol Renal Physiol 2020; 319:F335-44.

[44] Liu T, Yang Q, Zhang X, Qin R, Shan W, Zhang H, et al. Quercetin alleviates kidney fibrosis by reducing renal tubular epithelial cell senescence through the SIRT1/PINK1/mitophagy axis. Life Sci 2020; 257:118116.

[45] Elmansi AM, Awad ME, Eisa NH, Kondrikov D, Hussein KA, Aguilar-Pérez $A$, et al. What doesn't kill you makes you stranger: Dipeptidyl peptidase-4 (CD26) proteolysis differentially modulates the activity of many peptide hormones and cytokines generating novel cryptic bioactive ligands. Pharmacol Ther 2019; 198:90-108. 